\title{
Impacto de Grupos de Educação em Saúde na Qualidade de Vida de Hipertensos e Diabéticos
}

\author{
Raquel Missio ${ }^{1}$, Tiago Bittencourt de Oliveira ${ }^{2}$, Carlos Kemper ${ }^{3}$
}

\begin{abstract}
RESUMO
Avaliou-se o impacto dos grupos de educação em saúde na qualidade de vida de pacientes hipertensos e diabéticos. Este estudo foi composto por pacientes hipertensos e diabéticos da cidade de Santo Ângelo-RS, participantes de grupos de educação em saúde realizados por bolsistas do projeto PET/Vigilância em Saúde da Universidade Regional Integrada de Santo Ângelo-RS. A coleta de dados foi qualitativa-quantitativa e ocorreu por meio do questionário elaborado pelos autores e outro questionário, padronizado, sobre a qualidade de vida, chamado WHOQOL-bref, aplicados no início da realização dos grupos (até o terceiro encontro) e após seis meses de realização dos mesmos. Os dados foram analisados estatisticamente por meio do Teste não paramétrico de Wilcoxon. Foram entrevistados 9 indivíduos, sendo $66,7 \%$ do sexo masculino, com idade acima dos 51 anos e escolaridade com predomínio entre 5 a e $8^{a}$ séries (44,4\%). Cerca de 55,5\% eram hipertensos e diabéticos e usavam preferencialmente o SUS $(77,8 \%)$ como local de controle de suas patologias. Em relação aos domínios do WHOQOL-bref, o domínio psicológico apresentou mudança $(p=0,041)$, sendo esta negativa, após as atividades de educação em saúde. De acordo com os resultados, observou-se que as atividades de educação em saúde não provocaram mudanças significantes, exceto no domínio psicológico, que apresentou mudança negativa. Esta falta de modificações pode ser em razão de alguns fatores, como período relativamente curto de trabalho, baixa escolaridade e faixa etária elevada do grupo. Mesmo assim, observou-se que, no geral, os indivíduos avaliados relataram uma percepção positiva às atividades de educação e em relação a sua qualidade de vida.
\end{abstract}

Palavras-chave: Qualidade de vida. Educação para a saúde. WHOQOL-bref. Hipertensão. Diabetes Mellitus.

IMPACT OF EDUCATION GROUPS IN HEALTH ON THE QUALITY OF LIFE ON HIPERTENSIVE AND DIABETIC

\section{ABSTRACT}

Evaluated the impact of health education groups in the quality of life of hypertensive and diabetic patients. This study consisted of hypertensive and diabetic patients from the city of Santo Angelo-RS, participants in health education groups conducted by fellows of the PET Project - Health Surveillance at the University of Santo Angelo. The production of data was qualitative-quantitative and applied to evaluated authors in the evaluation of authors and another question, a standardized questionnaire of quality of life called WHOQOL-bref, applied at the beginning of realization of the groups (up to the third meeting) and after 6 months of realization. Data were statistically analyzed using the non-parametric Wilcoxon test. They interviewed nine individuals, $66.7 \%$ were male, over the age of 51 years and schooling predominantly between the 5th-8th grade (44.4\%). Approximately $55.5 \%$ were hypertensive and diabetic and used preferably SUS $(77.8 \%)$ and local control their diseases. Regarding the WHOQOL-bref domains, the psychological domain had change $(p=0.041)$, this negative being, after the health education activities. According to the results, it was observed that health education activities did not cause significant changes, except in the psychological domain that showed a negative change, this lack of change may be due to factors such as relatively short working hours, low education and high age group. Still, it was observed that the evaluated subjects reported a positive perception as educational activities and of their quality of life.

Keywords: Quality of life. Health education. WHOQOL-bref. Hypertension. Diabetes Mellitus.

Recebido em: 16/1/2016

Revisões requeridas em: 30/1/2018

Aceito em: 9/8/2018

\footnotetext{
Graduanda do Curso de Farmácia, da Universidade Regional Integrada do Alto Uruguai e das Missões - URI. Bolsista PET- Vigilância em Saúde do Ministério da Saúde.missio.raquel@gmail.com

2 Docente do curso de Farmácia da URI - Santo Ângelo, RS. Departamento de Ciências da Saúde. tiagofarm@gmail.com

3 Docente do curso de Educação Física da URI - Santo Ângelo, RS. Departamento de Ciências da Saúde.ckemper@san.uri.br
} 


\section{INTRODUÇÃO}

O conceito de qualidade de vida (QV) começou a ser desenvolvido em meados da década de 70, a partir de interesses que surgiram nas áreas das ciências sociais, econômicas, humanas e da saúde (CHAZAN; CAMPOS, 2013), mas somente na década de 90, a Organização Mundial de Saúde (OMS) propôs um conceito subjetivo, multidimensional, com aspectos positivos e negativos para definir qualidade de vida. Assim, a expressão é conceituada como "a percepção do indivíduo de sua posição na vida, no contexto da cultura e sistema de valores nos quais ele vive e em relação aos seus objetivos, expectativas, padrões e preocupações" (TAVARES; CÔRTES; DIAS, 2010).

Atualmente, os estudos sobre qualidade de vida têm se mostrado importantes em muitas áreas, como no campo da saúde, pois permite avaliar o impacto físico e psicossocial que a doença causa na vida do indivíduo, manifestado por ele mesmo (QUADROS, 2013).

No Brasil, pesquisas estão sendo realizadas para avaliar a qualidade de vida de pacientes portadores de doenças crônicas não transmissíveis, como hipertensão arterial sistêmica (HAS) e diabetes mellitus (DM), com o intuito de auxiliar no desenvolvimento de eficientes estratégias terapêuticas (BAMPI; GUILHEM; LIMA, 2008), evitando agravos, hospitalização e consequentes gastos públicos (MIRANZI et al., 2008).

Para isso, a Organização Mundial da Saúde (OMS) elaborou o questionário World Health Organization Quality of Life - 100 (WHOQOL-100) e a versão abreviada, WHOQOL-Bref, como instrumentos genéricos que podem ser usados para avaliar a qualidade de vida de indivíduos sadios ou com alguma patologia (LACERDA et al., 2011).

A qualidade de vida de pacientes hipertensos e diabéticos pode ser reestabelecida por meio de grupos de educação em saúde que auxiliam no acompanhamento e na orientação do controle dos níveis de pressão arterial e glicemia, pois estimulam a autonomia dos sujeitos envolvidos quanto à escolha da adoção de hábitos saudáveis, permitindo um melhor monitoramento de suas patologias e evitando agravos relacionados (OLIVEIRA et al., 2013), bem como propiciam um espaço de humanização, inclusão social e participação cidadã (PEREIRA; VIEIRA; FILHO, 2011).

Desta forma, grupos de educação em saúde, constituídos a partir da troca de conhecimentos dos profissionais de saúde e dos participantes, permite que ambos aprendam e ensinem reciprocamente (PEREIRA; VIEIRA; FILHO, 2011), favorecendo a comunicação e o desenvolvimento de ações educativas (MEN-
DONÇA; NUNES, 2014). As atividades de educação em saúde devem ser dialéticas e ir ao encontro das necessidades dos usuários. O modelo dialógico, fortalecendo a cidadania, considerando o ser como "singular", livre e participante de suas aprendizagens, é uma estratégia importante e faz com que profissionais e usuários aprendam com o colóquio educativo (FONTANA; BRUM; SANTOS, 2013).

O presente trabalho teve como objetivo avaliar o impacto dos grupos de educação em saúde na qualidade de vida de pacientes hipertensos e diabéticos.

\section{MÉTODO}

Foi realizado um estudo prospectivo e experimental qualitativo e quantitativo, composto por nove pacientes hipertensos e diabéticos participantes de grupos de educação em saúde, que se encontram semanalmente em quatro bairros da cidade de Santo Ângelo.

Os grupos eram coordenados por bolsistas do Projeto PET/Vigilância em Saúde, composto por acadêmicas do curso de farmácia, enfermagem, educação física e psicologia da Universidade Regional Integrada do Alto Uruguai e das Missões-URI, Campus Santo Ângelo.

Os conteúdos abordados nos grupos estavam relacionados com alimentação saudável, autoestima, uso correto de medicamentos e plantas medicinais, exercícios físicos, autocuidado com os pés, entre outros. A metodologia utilizada nos encontros era baseada em rodas de conversa, dinâmicas e apresentações de vídeos e imagens em Power Point, com o intuito de proporcionar um momento em que os participantes pudessem trocar informações e adquirir novos conhecimentos, sempre com o objetivo de orientar no controle dos níveis pressóricos e glicêmicos e, assim, evitar possíveis complicações para os pacientes.

Salienta-se que no início da realização das atividades verificava-se a pressão arterial e a glicemia dos participantes. Esses valores eram sempre repassados aos participantes, como também ficavam de posse dos coordenadores dos grupos para que pudessem acompanhar quaisquer alterações, podendo, assim, orientar os participantes quando fosse necessário.

Os critérios de inclusão para este estudo foi o diagnóstico de hipertensão arterial sistêmica e/ou diabetes mellitus, bem como a participação de forma regular aos grupos de educação em saúde, definida como a presença de, no mínimo, dois encontros dos quatro mensais, por, pelo menos, seis meses. Os par- 
ticipantes que se recusaram a assinar o Termo de Consentimento Livre e Esclarecido e os analfabetos foram excluídos deste trabalho.

Os dados foram coletados no mês de julho e novamente no mês de dezembro de 2014, por meio do questionário sociodemográfico desenvolvido pelos autores do estudo, que possibilitou a obtenção de informações como: gênero, idade, nível educacional, estado civil, patologia, local de controle. O questionário padronizado $W H O Q O L-b r e f$ específico para a aferição da qualidade de vida, desenvolvido pelo Grupo de Qualidade de Vida da Organização Mundial de Saúde (OMS) a partir do WHOQOL-100, foi uma versão abreviada deste (AZEVEDO et al., 2013).

O questionário WHOQOL-bref é formado por 26 questões, sendo as duas primeiras pertencentes ao domínio geral, que estão relacionadas com a autoavaliação do entrevistado sobre sua qualidade de vida e sua satisfação com a saúde. As demais 24 questões, estão divididas em 4 domínios, sendo o domínio físico (7 questões) psicológico (6) social (3) e ambiental (8) (CHAZAN; CAMPOS, 2013).

Desta forma, os domínios são definidos como à área do comportamento ou experiência que se deseja medir e estão representados por 24 facetas, sendo cada faceta composta por uma pergunta (CHAZAN; CAMPOS, 2013).

As respostas para todas as facetas foram estabelecidas para ser do tipo Likert, com escala de intensidade (nada/extremamente), capacidade (nada/completamente), frequência (nunca/sempre) e avaliação (muito insatisfeito/muito satisfeito; muito ruim/muito bom) (CHAZAN; CAMPOS, 2013). Os escores dos domínios foram transformados em uma escala linear que variou de 0-100, sendo estes respectivamente os valores menos e mais favoráveis de qualidade de vida.

O questionário elaborado pelos autores também era composto por perguntas abertas para que o entrevistado tivesse a possibilidade de falar sobre o tema. Foram anotados os relatos mais importantes e também utilizado um gravador para registrar as falas dos participantes, possibilitando, assim, o registro fidedigno das informações. As perguntas que nortearam o estudo foram: (1) Para o senhor (a), como foi participar de um grupo de educação em saúde?; (2) O que mudou na sua vida após participar do grupo de educação em saúde? Houve benefícios para a sua vida? Quais?; (3) Como o(a) senhor(a) avalia esse tipo de atividade?
Os dados qualitativos das entrevistas foram analisados mediante análise temática, que consiste em descobrir os núcleos de significado de uma comunicação. Foram percorridas as três etapas da análise temática: na pré-análise, o pesquisador teve "contato direto" com o material de campo, mediante leitura exaustiva e organização dos dados. No fim, o pesquisador desenvolveu "interpretações" a partir de fundamentação com literatura científica (MINAYO, 2010).

Nos relatos dos dados qualitativos foram preservados a identidade e o anonimatos dos sujeitos, adotando-se os codinomes P1, P2, P3, e assim sucessivamente para identificá-los. Os dados quantitativos foram organizados no programa Excel $2010^{\circledR}$ (Microsoft) e analisados em dados absolutos e porcentuais, sendo os resultados apresentados em tabelas. A avaliação estatística entre os escores dos domínios no início e após seis meses das atividades, bem como o resultado das questões $(1,2$ e 4$)$ pertencentes ao questionário WHOQOL-bref, foram analisados em programa estatístico, utilizando o Teste não paramétrico de Wilcoxon, considerando valor estatístico com significância de $p<0,05$. Para a avaliação dos escores foi necessário recodificar os valores das questões 3 , 4 e 26 , permitindo que ficassem com resultado final positivo.

Em relação aos aspectos éticos, no momento da entrevista, a pesquisadora e primeira autora do trabaIho explicou os objetivos do estudo, entregou o termo de Consentimento Livre e Esclarecido (TCLE) e, em seguida, solicitou que os participantes o assinassem se concordassem em participar da pesquisa. $O$ trabalho pertence ao projeto Qualidade e hábitos de vida de um grupo de hipertensos e diabéticos que fazem parte de um programa de educação em saúde aprovado pelo Comitê de Ética em Pesquisa (CEP) da URI - Campus Santo Ângelo, cujo número do parecer é 445.493 do dia 4 de novembro de 2013.

\section{RESULTADOS}

O estudo contou com a participação de nove indivíduos hipertensos e/ou diabéticos pertencentes ao grupo de educação em saúde, executado por bolsistas do Projeto PET/Vigilância em Saúde da Universidade Regional Integrada - URI -, campus Santo Ângelo. As informações referentes ao gênero, idade, nível educacional, estado civil, patologia e local de controle dos participantes foram adquiridas por meio do questionário sociodemográfico e estão apresentadas na Tabela 1 . 
Tabela 1 - Características gerais dos nove pacientes hipertensos e diabéticos

\begin{tabular}{lccc}
\hline & & $\mathbf{n}$ & $\%$ \\
\hline Gênero & Masculino & 6 & 66,7 \\
Idade & Feminino & 3 & 33,3 \\
& $51-60$ anos & 2 & 22,2 \\
Nível educacional & $61-70$ anos & 3 & 33,3 \\
& $>70$ anos & 4 & 44,4 \\
& 1a -4 a Série & 2 & 22,2 \\
Estado civil & 8a Série & 4 & 44,4 \\
& Ensino Médio completo & 2 & 22,2 \\
Patologia & Ensino Superior completo & 1 & 11,1 \\
& Solteiro ou separado & 4 & 44,4 \\
& Viúvo & 1 & 11,1 \\
Local de controle & Casado & 4 & 44,4 \\
& Hipertensão & 2 & 22,2 \\
& Diabetes Mellitus & 2 & 22,2 \\
& HAS* + DM** & 5 & 55,5 \\
& SUS & 7 & 77,7 \\
& Particular & 1 & 11,1 \\
& SUS e particular & 1 & 11,1 \\
\hline
\end{tabular}

*HAS: Hipertensão Arterial Sistêmica; **DM: Diabetes Melittus.

O questionário sociodemográfico também permitiu a avaliação de quesitos como o seguimento da dieta, expostos na Tabela 2.

Tabela 2 - Características referentes ao seguimento da dieta e prescrição médica dos pacientes hipertensos e diabéticos no início do estudo e após seis meses (fim)

\begin{tabular}{llcccc}
\hline & & \multicolumn{2}{c}{ INÍcIO } & \multicolumn{2}{c}{ FIM } \\
& & $\mathbf{n}$ & $\%$ & $\mathbf{n}$ & $\%$ \\
\hline \multirow{3}{*}{ Segue dieta } & Totalmente & 1 & 11,1 & 4 & 44,4 \\
& Parcialmente & 6 & 66,7 & 3 & 33,3 \\
& Não segue & 2 & 22,2 & 2 & 22,2 \\
\hline
\end{tabular}

A qualidade de vida estimada por intermédio do questionário WHOQOL-bref, possibilitou a avaliação, por meio de escores, de quesitos físicos, psicológicos, sociais e ambientais, sendo o maior escore obtido no início do estudo no domínio psicológico $(75,46$ $\pm 12,58$ ) e, ao término do trabalho, no domínio social $(70,37 \pm 12,58)$, em uma escala que varia de zero a 100 . A partir da obtenção dos escores destes domínios, obteve-se a estimativa para o cálculo do escore geral dos participantes, sendo no início e o no fim, respectivamente, de 74,73 $( \pm 7,03)$ e 73,36 $( \pm 6,96)$ (Tabela 3$)$.
Tabela 3 - Escores por domínio a partir do questionário WHOQOL-bref dos pacientes hipertensos e diabéticos estudados no início do estudo e após seis meses (fim)

\begin{tabular}{cccccc}
\hline & INÍCIO & DP* & FIM & DP & p \\
\hline Domínio Físico (0-100) & 62,30 & $\pm 11,59$ & 56,75 & $\pm 12,56$ & 0,073 \\
Domínio Psicológico (0-100) & 75,46 & $\pm 12,58$ & 68,52 & $\pm 8,62$ & 0,041 \\
Domínio Social (0-100) & 68,52 & $\pm 10,85$ & 70,37 & $\pm 12,58$ & 0,414 \\
Domínio Ambiental (0-100) & 64,93 & $\pm 11,77$ & 65,63 & $\pm 12,40$ & 0,865 \\
Domínio Geral (0-100) & 74,73 & $\pm 7,03$ & 73,36 & $\pm 6,96$ & 0,214 \\
\hline *DP: Desvio Padrão. p apresenta significância estatística \\
\multicolumn{5}{c}{ quando p<0,05. }
\end{tabular}

Na Tabela 4 foi possível observar que, em ambas as análises, a maioria dos participantes considerou sua qualidade de vida "boa" $(77,8 \%)$, seguido de $22,2 \%$ que consideraram "muito boa".

Tabela 4 - Avaliação da qualidade de vida dos participantes no início do estudo e após seis meses (fim) (Questão 1 do questionário WHOQOL-bref)

\begin{tabular}{cccc}
\hline AVALIAÇÃO & $\begin{array}{c}\text { INÍCIO } \\
\mathbf{n}(\%)\end{array}$ & $\begin{array}{c}\text { FIM } \\
\mathbf{n}(\%)\end{array}$ & $\mathbf{p}$ \\
\hline Muito ruim & $0(0)$ & $0(0)$ & \\
Ruim & $0(0)$ & $0(0)$ & \\
Nem ruim nem boa & $0(0)$ & $0(0)$ & 1,00 \\
Boa & $7(77,8)$ & $7(77,8)$ & \\
Muito boa & $2(22,2)$ & $2(22,2)$ & \\
\hline
\end{tabular}

Os relatos sobre como foi participar dos grupos de educação em saúde pelos pacientes foram registrados:

"Ah foi bom, esclarecedor, a gente tira muita dúvida, coisas que jamais imaginei. Muitas vezes a gente ocorre um problema de saúde porque a gente não tem uma orientação. Aí eu esclareci muitas dúvidas. 'Tô' seguindo as orientações de vocês" (P5).

"Antes não existia, agora tá acontecendo e tá bom. E eu gosto muito; não venho só quando não posso" (P6).

Também os pacientes relataram mudanças com a intervenção realizada nos grupos e avaliaram as atividades realizadas:

"O que mudou é que eu me controlei e melhorei da diabetes, podia tá muito pior, melhorei grande coisa. Benefícios? Ih, aí eu me cuidei. Quando eu cheguei aqui, eu 'tava' com 330 de diabetes e agora 'tô' com 108. É uma grande coisa. A pressão tá boa" (P3). 
"Olha mudou na minha vida, que eu tenho uma coisa para me distrair quarta-feira que 'tô' sempre aqui. Eu gosto de vir, já que eu moro sozinho né e aqui é um entretimento pra mim. Vê tudo e explicarem tudo pra mim. Eu vejo que pra mim é um passatempo. Benefícios: 'tô' mais alegre, mais vivido, mais melhor" (P4).

“Benefícios: 'tô' me desenvolvendo mais, a gente fica muito sozinha, assim a gente vai no grupo para conversar e ainda aprender as coisas" (P6).

“Eu acho que é muito bom né, porque a gente tem mais conhecimento das coisas, como é. Porque antes a gente muitas vezes não valorizava muito né, assim, como que é tal coisa, como tu pode se cuidar, o que tu pode usar para melhorar na tua saúde. Os exercícios também né. Então tudo isso foi muito proveitoso. O conteúdo era muito bem passado. Eu tenho agradecer a vocês" (P2).

"Acho muito bom, a gente está se desenvolvendo, aprendendo muita coisa na saúde pra tudo né desenvolvendo até na memória, eu tenho problema na memória né" (P6).

“Boa, como excelente. Vocês já estão fazendo um trabalho acadêmico e ao mesmo tempo prestando um serviço à sociedade. Esse programa tem que intensificar, é muito bom. E vocês vêm fazendo isso bem, com sucesso, tranquilo, tendo paciência, às vezes tem uns mais devagar. Vocês têm tido a paciência necessária" (P7).

Em relação à satisfação com a saúde $55,6 \%$ dos entrevistados consideraram-se "satisfeitos" nos dois momentos estudados (Tabela 5).

Tabela 5 - Satisfação com a saúde dos participantes no início do estudo e após seis meses (fim) (Questão 2 do questionário WHOQOL-bref).

\begin{tabular}{cccc}
\hline COMO SE SENTE & $\begin{array}{c}\text { INÍCIO } \\
\mathbf{n}(\%)\end{array}$ & $\begin{array}{c}\text { FIM } \\
\mathbf{n}(\%)\end{array}$ & $\mathbf{p}$ \\
\hline Muito insatisfeito & $0(0)$ & $0(0)$ & \\
Insatisfeito & $1(11,1)$ & $2(22,2)$ & \\
Nem satisfeito nem insatisfeito & $3(33,3)$ & $1(11,1)$ & 0,564 \\
Satisfeito & $5(55,6)$ & $5(55,6)$ & \\
Muito satisfeito & $0(0)$ & $1(11,1)$ & \\
\hline
\end{tabular}

No domínio físico, no que se refere à necessidade de algum tratamento médico, no início do estudo $44,4 \%$ dos participantes acreditavam que era "muito pouco" necessário algum tratamento médico para levar a vida diária, entretanto, após 6 meses, 66,7\% passaram a acreditar ser preciso algum tratamento (Tabela 6).
Tabela 6 - Necessidade de algum tratamento médico para levar a vida diária no início do estudo e após seis meses (fim) (Questão 4 do questionário WHOQOL-bref)

\begin{tabular}{cccc}
\hline $\begin{array}{c}\text { NECESSIDADE DE TRATAMENTO } \\
\text { MÉDICO }\end{array}$ & $\begin{array}{c}\text { INÍCIO } \\
\mathbf{n}(\%)\end{array}$ & $\begin{array}{c}\text { FIM } \\
\mathbf{n}(\%)\end{array}$ & $\mathbf{p}$ \\
\hline Nada & $1(11,1)$ & $2(22,2)$ & \\
Muito pouco & $4(44,4)$ & $6(66,7)$ & \\
Mais ou menos & $3(33,3)$ & $1(11,1)$ & 0,059 \\
Bastante & $1(11,1)$ & $0(0)$ & \\
Extremamente & $0(0)$ & $0(0)$ & \\
\hline
\end{tabular}

\section{DISCUSSÃO}

No presente estudo observou-se que a maioria dos participantes era do sexo masculino $(66,7 \%)$, sendo este dado divergente de outras pesquisas com pacientes hipertensos e/ou diabéticos encontrados na literatura. De acordo com o estudo realizado no mesmo município de Santo Ângelo (DAHMER et al., 2015), $68 \%$ dos indivíduos participantes eram do sexo feminino, dado este também encontrado em Uberaba - MG -, onde $69,6 \%$ dos participantes eram mulheres (TAVARES; CÔRTES; DIAS, 2011).

A incidência das doenças crônicas não transmissíveis está associada a fatores como idade, escolaridade e estado civil, ou seja, as prevalências destas patologias são maiores em indivíduos entre 50-59 anos e acima de 60 anos, com menor escolaridade, nos sujeitos solteiros e naqueles divorciados/separados/viúvos (BRISCHILIARI et al., 2014). Neste trabalho observou-se um predomínio de indivíduos com faixa etária acima dos 61 anos (77,7\%), com o nível educacional entre 5 a e 8 a série $(44,4 \%)$ e, em relação ao estado civil, $44,4 \%$ eram solteiros ou separados.

A hipertensão arterial sistêmica é duas vezes mais frequente em pessoas acometidas pelo Diabetes Mellitus (TAVARES; Côrtes; Dias, 2010). Neste sentido, observou-se que $55,5 \%$ dos participantes possuíam ambas as patologias. Em relação ao local de controle, $77,7 \%$ relataram usar o Sistema Único de Saúde (SUS). Isto pode ser explicado pelo fato de que o SUS possui o seu modelo de atenção à saúde baseado nas Estratégias Saúde da Família (ESF) (AZEVEDO et al., 2013), que, em relação às doenças crônicas, tem o objetivo de prevenir, controlar e reabilitar (MENDONÇA; NUNES, 2014).

Em relação ao quesito seguimento da dieta, presente no questionário sociodemográfico, observou-se que, no início das atividades em grupo, 1 $(11,1 \%)$ paciente seguia totalmente a dieta e, após as 
atividades de educação em saúde, 4 (44,4\%) pacientes passaram a seguir "totalmente" a dieta. Esta modificação está intimamente ligada a questões culturais e econômicas dos indivíduos, sendo de difícil mudança, no entanto ações educativas desenvolvidas em grupos de educação em saúde sobre este tema criam uma ótima possibilidade de adoção de hábitos alimentares saudáveis, pois estimulam o autocuidado do indivíduo (OLIVEIRA et al., 2013).

Quanto aos dados dos domínios do WHOQOL-bref, observou-se que o domínio físico, em escala de zero a 100, no início e no final das atividades, apresentou menor valor, sendo eles, respectivamente, 62,30 e 56,75 . De acordo com os pesquisadores, os portadores de doenças crônicas podem ser afetados fisicamente pelo fato de necessitarem usar medicamentos diariamente, além de precisarem um acompanhamento assistencial contínuo, em razão da cronicidade destas patologias (TAVARES; CÔRTES: DIAS, 2010). Esta diferença, entretanto, não é considerada significante, mas apresenta uma tendência à redução.

Por outro lado, o maior escore no início dos grupos de educação em saúde foi observado no domínio psicológico $(75,46)$, que avalia questões relacionadas com sentimentos positivos, concentração, autoestima, aparência, sentimentos negativos, espiritualidade, religião e crenças pessoais, porém, após seis meses das atividades, diminui para 68,52 , sendo esta diminuição considerada significante.

De acordo com um estudo que avaliou os efeitos de um Programa de Promoção do Envelhecimento Saudável na Qualidade de Vida dos Idosos durante um ano, realizado no Ambulatório de Geriatria do Hospital das Clínicas da FMUSP, os resultados para o domínio psicológico no início e no fim foram, respectivamente, 66,41 e 70,08 (TAMAl, 2010). Esta divergência entre os resultados dos dois estudos pode ser explicada pelo menor tempo de atividades de educação em saúde realizadas por este estudo, bem como pelo fato de que a qualidade de vida pode ser influenciada por aspectos culturais, religiosos, éticos e valores pessoais (SANTOS; MOREIRO; RODRIGUES, 2008), e, por isso, pode sofrer alteração no decorrer do tempo (BAMPI; GUILHEM; LIMA, 2008).

Em relação ao domínio social e ambiental, não houve alteração significante durante o período estudado. A participação em grupos de educação em saúde permite a troca de saberes, diálogo, inclusão dos usuários e construção de conhecimentos, favorecendo as relações pessoais que contribuem positivamente na qualidade de vida de pessoas hipertensas e diabéticas (MENDONÇA; NUNES, 2014). Quanto ao domínio ambiental, que está relacionado ao ambiente em que a pessoa vive, integrando desde aspectos físicos até oportunidade de lazer, situação financeira e condições de transporte, o resultado obtido mostrou-se positivo. Acredita-se que, mesmo após a descoberta da doença, os gastos financeiros relacionados ao tratamento não abalaram as oportunidades de lazer e entretenimento, bem como entende-se que o ambiente em que vivem e o meio de transporte que utilizam lhes satisfazem (AZEVEDO et al., 2013).

No que diz respeito ao domínio geral, o resultado no início e após as atividades de educação não apresentaram alterações significantes. Salienta-se que, em ambos os momentos estudados, os valores dos domínios (social, físico, psicológico e ambiental) apresentaram valores acima de 50. Assim, avalia-se que os indivíduos entrevistados mostraram uma percepção positiva para a qualidade de vida (MIRANZI et al., 2008), pois, mesmo com uma diminuição do domínio psicológico, somente este dado não foi capaz de afetar a qualidade de vida destes pacientes.

Deve-se destacar que usamos nos grupos de educação o ensinado por Paulo Freire, de que a problematização na educação popular em saúde é uma forma de incitar para a discussão e apreensão de saberes. Esta situação empodera os sujeitos por meio do conhecimento e torna-os cidadãos livres para escolher e deliberar, tornando-os coprodutores de sua saúde, evoluindo da posição de "paciente" para a posição de sujeito crítico e ativo no seu processo de cuidado (FREIRE, 1983; FERNANDES; BACKES, 2010).

$A$ atividade de educação realizada possivelmente favoreceu a consciência crítica dos participantes, pois oportunizou manifestar dificuldades de acesso, omissões e resolutividade no processo de produção de saúde do serviço. Para que ocorra transformação na realidade, é imprescindível harmonizar espaços de reflexão individual e coletiva (FERNANDES; BACKES, 2010).

Destaca-se que a maioria dos participantes dos grupos de educação desenvolvidos pelo PET/VS, e os participantes deste estudo, são idosos. Por isso, alguns relataram que os grupos são um motivo para sair de seus lares. Outro trabalho certifica o encontrado (TAHAN; CARVALHO, 2010), visto que fica evidente nas falas que a participação no grupo é considerada uma atividade de lazer, pois eles mantêm contato com os outros participantes.

Os grupos de promoção da saúde devem atuar além do tema doença no campo comunitário, abrindo possibilidades para as ciências da saúde e do homem, com atividades que vão além do simples objetivo de 
combater as doenças das pessoas. Estas deverão ser somadas à tarefa da preocupação com a própria identidade da pessoa humana na busca do grau mais elevado possível de saúde física, mental e social para si e para a sociedade (FIGUEIREDO; RODRIGUES NETO; LEITE, 2012).

Após participarem dos grupos, os pacientes relataram que começaram a cuidar mais de sua saúde. Infere-se que os participantes dos grupos de educação sentem-se mais capacitados para se cuidarem, uma vez que já conhecem as complicações e os riscos das suas doenças, estando aptos, portanto, à promoção de sua própria saúde. Os grupos de promoção à saúde são realizados para que instrumentalizem os seus participantes ao serviço da autonomia e do desenvolvimento contínuo do nível de saúde e, consequentemente, melhoria da qualidade de vida (FIGUEIREDO; RODRIGUES NETO; LEITE, 2012).

Em relação à questão 1 , que diz respeito à avaliação da qualidade de vida, e a questão 2, que menciona a satisfação do participante referente a sua saúde, nos dois momentos do estudo a maioria considerou "boa" $(77,8 \%)$ sua qualidade de vida e "satisfeitos" (55,6\%) em relação a sua saúde. Estes dados permitem perceber que, mesmo estes indivíduos estando acometidos por doenças que requerem tratamento $\mathrm{e}$ acompanhamento permanente, isto não os impedem de considerarem sua qualidade de vida boa e estarem satisfeitos com sua saúde.

Na questão número 4, pertencente ao domínio físico, que se refere à necessidade de algum tratamento médico para levar a vida diária, observou-se que ocorreu um aumento de $11,1 \%$ para $22,2 \%$ nos participantes que passaram a avaliar como "nada" a necessidade de algum tratamento médico para levar a vida diária, e de $44,4 \%$ para $66,7 \%$, nos participantes que passaram a avaliar como "muito pouco". Esta alteração, entretanto, não é significante, mesmo assim ocorreu uma tendência de mudança nos resultados, que pode ser explicada pelo fato de que as atividades realizadas em grupos de educação em saúde, estimulando a autonomia e favorecendo o autocuidado, consequentemente auxiliam no controle dos níveis pressóricos e glicêmicos, permitindo que os sujeitos envolvidos consigam controlar suas patologias (SANTOS; MOREIRO; RODRIGUES, 2008). Ressalta-se, também, que grupos de saúde formados por equipe multiprofissional possibilitam a valorização e a busca por outros profissionais da saúde, como farmacêuticos, enfermeiros, nutricionistas, psicólogos, educadores físicos, entre outros, descentralizando a figura do médico como o único cuidador da sua saúde (SILVA et al., 2013).

\section{CONCLUSÕES}

Os resultados obtidos após seis meses das atividades de educação em saúde não apresentaram melhora significante no questionário WHOQOL-bref. A redução no domínio psicológico pode ser por alguns fatores, como período relativamente curto de trabalho, nível educacional baixo, o que atrapalha a interpretação das informações transmitidas, e faixa etária elevada, dificultando a mudança nos hábitos de vida. Salienta-se que as atividades de intervenção não tiveram o objetivo específico de modificar as questões que contemplam o questionário WHOQOL-bref e, sim, trazer conhecimento sobre as patologias e melhoria do bem-estar dos participantes. Mesmo assim, observou-se que os indivíduos avaliados apresentam uma percepção positiva em relação a sua qualidade de vida e, em seus relatos, demonstraram satisfação com as atividades de educação e melhoria de seus parâmetros clínicos.

\section{REFERÊNCIAS}

AZEVEDO, A. L. S. et al. Doenças crônicas e qualidade de vida na atenção primária à saúde. Cad. Saúde Pública, São Paulo, v. 29, n. 9, p. 1.774-1.782, set. 2013.

BAMPI, L. N. S.; GUILHEM, D.; LIMA, D. D. Qualidade de vida em pessoas com lesão medular traumática: um estudo com o WHOQOL-bref. Rev. Bras. Epidemiol., São Paulo, v. 11, n. 1, p. 67-77, mar. 2008.

BRISCHILIARI, S. C. R. et al. Doenças crônicas não transmissíveis e associação com fatores de risco. Rev. Bras. Cardiol., Rio de Janeiro, v. 27, n. 1, p. 35-42, jan./fev. 2014.

CHAZAN, A. C. S.; CAMPOS, M. R. Qualidade de vida de estudantes de medicina medida pelo WHOQOL-bref - Uerj, 2010. Rev. Bras. Educ. Med., Rio de Janeiro, v. 37, n. 3, p. 376-384, jul./set. 2013.

DAHMER, L. et al. Avaliação da qualidade de vida de pacientes hipertensos e diabéticos. Rev. Cont. Saud., ljuí, v. 15, n. 28, p. 41-49, jan./jun. 2015.

LACERDA, S. M. et al. Qualidade de vida de idosos atendidos em Programa de Assistência Domiciliária. Rev. Bras. Geriatr. Gerontol., Rio de Janeiro, v. 14, n. 2, p. 329-342, 2011.

FERNANDES, M. C. P.; BACKES, V. M. S. Educação em saúde: perspectivas de uma equipe da Estratégia Saúde da Família sob a óptica de Paulo Freire. Rev. Bras. Enferm., v. 63, n. 4, p. 567-573, 2010.

FIGUEIREDO, M. F. S.; RODRIGUES NETO, J. F.; LEITE, M. T. S. Educação em saúde no contexto da Saúde da Família na perspectiva do usuário. Interface Comum. Saúde Educ., Botucatu, v. 16, n. 41, p. 315-332, 2012.

FONTANA, R. T.; BRUM, Z. P.; SANTOS, A. V. Health education as a strategy for healthy sexuality. Rev. Pesqui. Cuid. Fundam. (on-line), Rio de Janeiro, v. 5, n. 4, p. 529-36, 2013. FREIRE, P. Pedagogia do oprimido. 12. ed. Rio de Janeiro: Paz e Terra, 1983. 
MENDONÇA, F. F.; NUNES, E. F. P. A. Atividades participativas em grupos de educação em saúde para doentes crônicos. Cad. Saúde Coletiva, Rio de Janeiro, v. 22, n. 2, p. 200204, jun. 2014.

MINAYO, M. C. S. O desafio do conhecimento: pesquisa qualitativa em saúde. 12. ed. São Paulo, SP: Hucitec, 2010.

MIRANZI, S. S. C. et al. Qualidade de vida de indivíduos com diabetes mellitus e hipertensão acompanhados por uma equipe de saúde da família. Texto Contexto Enferm., Florianópolis, v. 17, n. 4, p. 672-679, out./dez. 2008.

OLIVEIRA, T. L. et al. Eficácia da educação em saúde no tratamento não medicamentoso da hipertensão arterial. Acta Paul. Enferm., São Paulo, v. 26, n. 2, p. 179-184, 2013.

PEREIRA, A. V.; VIEIRA, A. L. S.; FILHO, A. A. Grupos de educação em saúde: aprendizagem permanente com pessoas soropositivas para o HIV. Rev. Trab. Educ. Saúde, Rio de Janeiro, v. 9, n. 1, p. 25-41, mar./jun. 2011.

QUADROS, A. S. Vivendo mais e melhor: qualidade de vida relacionada à saúde. Rev. Bras. Cardiol. Invasiva, São Paulo, v. 21, n. 1, p. 5-6, jan./mar. 2013.
SANTOS, M. C.; MOREIRO, F. C. F. S.; RODRIGUES, M. R. Estudo sobre qualidade de vida com pacientes pós-TMO: aplicação do questionário WHOQOL-Bref. Rev. O Mundo da Saúde, São Paulo, v. 32, n. 2, p. 146-156, abr./jun. 2008.

SILVA, E. P. et al. Projeto terapêutico singular como estratégia de prática da multiprofissionalidade nas ações de saúde. Rev. Bras. Ciên. Saúde, v. 17, n. 2, p. 197-202, 2013.

TAHAN, J.; CARVALHO, A. C. D. Reflexões de idosos participantes de grupos de promoção de saúde acerca do enveIhecimento e da qualidade de vida. Saúde Soc., v. 19, n. 4, p. 878-88, 2010.

TAMAI, S. A. B. Avaliação de um programa de educação em saúde na qualidade de vida e no estado de bem-estar em idosos. 2010. Tese (Doutorado em Patologia) - Faculdade de Medicina da Universidade de São Paulo - USP, Programa de Patologia, São Paulo, 2010.

TAVARES, D. M. S.; CÔRTES, R. M.; DIAS, F. A. Qualidade de vida e comorbidades entre idosos diabéticos. Rev. Enferm Uerj, Rio de Janeiro, v. 18, n. 1, p. 97-103, jan./mar. 2010.

. Qualidade de vida de idosos com Diabetes Mellitus.

Ciênc. Cuid. Saúde, v. 10, n. 2, p. 290-297, abr./jun. 2011. 